\title{
Effects of Multiple Extrusions on Structure-property Performance of Natural Fiber High- density Polyethylene Biocomposites
}

\author{
Elham Nadali ${ }^{a}$, Mohammad Layeghi, Ghanbar Ebrahimi ${ }^{b}$, Reza Naghdi ${ }^{*}$, Mehdi Jonoobi ${ }^{b}$,
} Mohammah Mehdi Khorasanic, Yaser Mirbagheri ${ }^{d}$

\author{
${ }^{a}$ Department of Wood and Paper Science and Technology, Faculty of Natural Resources, Semnan \\ University, Iran \\ ${ }^{b}$ Department of Wood and Paper Science and Technology, Faculty of Natural Resources, \\ University of Tehran, Iran \\ ${ }^{c}$ Petrochemical Research and Technology Company (NPC-rt), National Petrochemical Company (NPC), \\ Tehran, Iran \\ ${ }^{d}$ Department of Mechanical Engineering, Islamic Azad University, Golpayegan Branch, Iran
}

Received: March 18, 2017; Revised: September 18, 2017; Accepted: November 27, 2017

Detailed analysis of the effects of multiple extrusions on physical, mechanical, micro-structural as well as dynamic mechanical thermal properties of natural fiber high-density polyethylene (HDPE) composites is reported. Composite materials containing HDPE, wood flour, and Maleic Anhydride polyethylene (MAPE) were manufactured and subjected to a recycling process consisting of up to four times grinding and reprocessing under industrial conditions. A wide range of analytical methods including bending tests, modulus of elasticity, impact strength, Scanning electron microscopy (SEM), fiber length measurement, water absorption tests, and dynamic mechanical thermal analysis (DMTA) were employed to understand the effects of recycling on natural fiber-HDPE composites. The results revealed that the recycled composites had lower bending strength and modulus of elasticity values, as compared to the reference counterparts. Also, the once recycled composites showed higher impact strength. Results, as well, indicated that generally the recycled composites had lower water absorption values as compared to the reference ones. The results obtained from DMTA exhibited a decrease in storage modulus and an increase in mechanical loss factor $(\tan \delta)$ for all composites subjected to recycling. Alterations in phase transition temperatures and intensities were also monitored and the possible reasons were analyzed.

Keywords: Biocomposites, recycling, mechanical properties, physical properties, DMTA.

\section{Introduction}

Natural fibers offer numerous benefits and challenges in fabrication of natural fiber plastic composites, besides their cost-effectiveness. ${ }^{1}$ One major advantage of such composites over the conventional thermosetting systems is their potential to be recycled either during the manufacturing process (internal recycling) or later after the service life (external recycling). This is an added advantage in terms of the ever-increasing environmental concerns, in particular, the problem of rapidly filling up landfills. However, many recycling processes also require extensive grinding as well as high-temperature mixing steps bringing about some unavoidable extent of degradation in the properties of the constituents and consequently the composite.

Over the last two decades, a large number of studies have been conducted underlining that sequential processing cycles impose a polymer matrix chain scission resulting from a thermal oxidation during reprocessing in both neat and reinforced

*e-mail: reza_naghdi@semnan.ac.ir polypropylene and polyethylene composites. This behavior is caused by polymer chain degradation due to harsh thermal and stress steps exerted in the course of recycling process. ${ }^{3}$ This thermal degradation can result on one hand in both cross-linking and chain scission of the polymer matrix and on the other hand in structural and chemical attrition of wood flour. ${ }^{4-13}$ The literature review indicates that PP is significantly less stable than PE. ${ }^{14}$ Although the trend is generally a loss of mechanical properties ${ }^{15-18}$, reprocessing can also lead to enhanced strength and stiffness values attributed to the improved dispersion of fiber within the polymer matrix. . $^{16,19-23}$ The archival publications indicate that water absorption and thickness swelling behavior of reprocessed natural fiber plastic composites depend on a numerous factors including interface quality, chemical composition, fiber length and distribution, and density. The results confirmed that generally the recycling process boosted water resistance and dimensional stability in the studied formulations. This is mainly due to better fiber distribution, higher density, lower amounts of voids, better interface quality, and improved hydrophobicity 
in the recycled composites. However, some other important variables such as processing system, temperature, fiber loading, and possible environmental impacts on physical properties were not included. ${ }^{2}$ Beg and Pickering (2008) studied the effects of reprocessing of wood fiber reinforced PP composites on their physical and mechanical properties. Tensile strength and Young's modulus reduced linearly from $41 \mathrm{MPa}$ and $4553 \mathrm{MPa}$ for the original composites to 31 and $3800 \mathrm{MPa}$, respectively, for those reprocessed 8 times. However, the failure strain increased as the composites were reprocessed more. Impact strength, flexural strength, and flexural modulus decreased with increased number of time the composite formulations were reprocessed. The change in mechanical properties was reported to be mostly arising from fiber fracture that occurred during reprocessing, with the subsequent reduction in average fiber length from 2.36 $\mathrm{mm}$ for the original fiber to $0.37 \mathrm{~mm}$ for the fiber extracted from the composites reprocessed for 8 times. ${ }^{16,18}$

Conventional static tests are usually employed to characterize the mechanical properties of such composites, which generally include tensile, bending, and impact tests. Since fiber-reinforced thermoplastic composites can undergo miscellaneous types of dynamic stresses during service, investigation on the dynamic mechanical properties of these materials calls for immediate attention. It should also be mentioned that dynamic mechanical properties depend on a range of factors such as type of fiber, fiber length and orientation, fiber loading, fiber dispersion, and fiber-matrix adhesion. $^{24}$

Dynamic mechanical thermal analysis (DMTA) is a sensitive technique that characterizes the mechanical responses of materials by monitoring property changes based on the temperature and/or frequency of oscillation. The technique separates the dynamic response of materials into two separate parts: an elastic part and a viscous one. The elastic process describes the energy stored in the system (storage modulus), while the viscous component describes the energy dissipated during the process (loss modulus). ${ }^{25-29}$ Both phases in a natural fiber-thermoplastic composite, reveal time-dependent properties. DMTA can provide invaluable information on the viscoelastic properties of the materials over a relatively short time. ${ }^{30}$ Thus, a deep and detailed understanding of dynamic mechanical properties of natural fiber thermoplastic composites is indispensable to assess the mechanical efficiency of the product. ${ }^{31}$ Studying the effect of natural fibers on thermal and mechanical properties of natural Fiber polypropylene composites through DMTA, Tajvidi et al. (2006) expressed an increase in storage and loss moduli and a decrease in the mechanical loss factor ( $\tan \delta$ ) for all composites indicating that the mentioned composites behaved more elastically compared to the pure PP counterparts. Results showed that glass transition was slightly shifted to lower temperatures in the given composites. ${ }^{24}$ In spite of the pertinence of the above-mentioned subjects, papers on composites recycling are rather scarce. Several investigations have focused on using recycled HDPE from different sources or virgin/recycled HDPE blends as matrices for composites containing postindustry pine wood flakes, Pinus radiata medium density fibers, sawmill waste wood flour, bagasse fibers, P. radiata waste sawdust, and maple wood flour, among others. ${ }^{32-37}$ On the other hand, investigations on composites recycling have been generally on PP-matrix composites containing flax fiber bundles, hemp, sisal, bleached softwood fibers, kenaf fibers, rice hulls, and wood flour. ${ }^{38-42}$ Evolution of mechanical properties of composites in the course recycling remains unknown in some cases. Whilst some papers report small reductions of composites tensile modulus and strength after recycling ${ }^{38}$ or maintained composite properties after reprocessing ${ }^{40-41}$, some others underscore increases in some mechanical properties, depending on the kind of cellulosic fibers used and on the number of reprocessing cycles undergone by the composites. ${ }^{18,21,39,43}$

The existing literature review denotes that several studies have been conducted on natural fiber recycled polymer composites, and some researchers have investigated on the external recycling of such material. To the best of the authors' knowledge, there is scanty information on the effects of reprocessing (internal recycling) cycles on structure-property performance of natural fiber high-density polyethylene composites. The present paper is a detailed investigation on the effects of reprocessing on physical, mechanical, and microstructural properties as well as on dynamic mechanical thermal analysis of natural fiber-HDPE composites. A wide range of characterization techniques has been employed to achieve better insight into possible reasons behind changes imposed by recycling.

\section{Materials and Methods}

\subsection{Materials}

High-density Polyethylene (HDPE ) was an extrusion grade with trade name EX5 (Maroon petrochemical company, Iran) with a melt flow index of $8 \mathrm{~g} / 10 \mathrm{~min}\left(2.16 \mathrm{~kg}, 190^{\circ} \mathrm{C}\right)$ and a density of $0.949 \mathrm{~g} / \mathrm{cm}^{3}$. An industrial grade MAPE (Maleic Anhydride grafted Polyethylene) was supplied by Karangin Chemicals Inc, Iran and used as compatibilizer. Wood flour (poplar) was obtained from grinding wood mill wastes in an industrial pulverizer. All lignocellulosic materials were then screened using an industrial shaker and fractions between 20 and 80 mesh were collected and dried in an air-circulating vertical drier at $140{ }^{\circ} \mathrm{C}$ for $5 \mathrm{~min}$ prior to use.

\subsection{Methods}

\subsubsection{Composites preparation and recycling process}

Composite formulations were produced as indicated in Table 1 . 
Table 1. Composites of evaluated formulations (wt $\%)$

\begin{tabular}{cccccc}
\hline Sample code & Fiber type & $\begin{array}{c}\text { HDPE content } \\
(\mathrm{wt} \%)\end{array}$ & Fiber content (wt\%) & $\begin{array}{c}\text { MAPE content } \\
(\mathrm{wt} \%)\end{array}$ & $\begin{array}{c}\text { Number of times } \\
\text { recycled }\end{array}$ \\
\hline WFPE Orig & Wood flour & 32 & 65 & 3 & 0 \\
WFPE Rec1 & Wood flour & 32 & 65 & 65 & 1 \\
WFPE Rec2 & Wood flour & 32 & 65 & 3 & 2 \\
WFPE Rec3 & Wood flour & 32 & 65 & 3 & 3 \\
WFPE Rec4 & Wood flour & 32 & & 3 & 4 \\
\hline
\end{tabular}

Composites materials containing $65 \mathrm{wt} \%$ fiber content, 32 $\mathrm{wt} \% \mathrm{HDPE}$, and $3 \mathrm{wt} \%$ MAPE were mixed at $140{ }^{\circ} \mathrm{C}$ in an industrial turbo mixer (Suzhou DYUN recycling machinery CO., LTD) at $200 \mathrm{rpm}$ for $10 \mathrm{~min}$ and stored in vacuum bags for further stages. The well-mixed composite materials were then transferred in to a counter rotating twin-screw extruder (Shanghai JWELL extrusion machinery CO. LTD) under the conditions given in Table 2.

Composite production involved at least 5 one-meter replications for each formulation. Recycling was performed in the following stages: first the composite profiles were ground into chips using an industrial crusher, and then the chips were pulverized and transferred again in to the extruder after drying. Reprocessing was carried out in the same mixer under the same mixing conditions.

Test specimens were cut out of these $12-\mathrm{mm}$ thick composite profiles. The recycling process was repeated 4 times with at least five replications for each formulation.

\subsubsection{Characterization}

After the first, second, third, and fourth cycles, samples were removed from the stream and then characterized. Original samples (not recycled) were also tested in the same way. Throughout this paper, the suffix Orig refers to original and suffixes Rec1, Rec2, Rec3, and Rec4 refer to one time, two time, three time, and four time recycled materials, respectively.

\subsubsection{Fiber length measurement}

A small amount of composite material prior to and after reprocessing was solubilized in boiling $P$-xylene (\%99.9), and then the fibers were separated from the viscous solution by acetone washing. Fiber length was measured using Nikon optical microscope (100X), and a total of 100 fibers were measured per composite formulation.

\subsubsection{Static tests}

Three-point bending tests were carried out according to ASTM D-790 specifications. The nominal dimensions of specimens were $300 \times 30 \times 12 \mathrm{~mm}$. The span was $20 \mathrm{~cm}$, and the crosshead speed was $5 \mathrm{~mm} / \mathrm{min}$. The tests were performed using an Instron testing machine model 5566 equipped with data acquisition system and a load-cell of $10 \mathrm{kN}$. Five specimens were tested from each group of specimens.

Unnotched Izod impact tests were conducted according to ASTM D-256 specifications. The tests were performed using a Ceast impact testing machine model 6958. The nominal dimensions of specimens were $65 \times 13 \times 12 \mathrm{~mm}$. The capacity of impact testing machine was $7.5 \mathrm{j}$, and 5 specimens were tested from each group of specimens.

\subsubsection{Physical tests}

The measurement of water absorption was conducted according to ASTM D-570 specifications, and five specimens were tested from each group. To do so, the specimens were first placed in an oven at $100^{\circ} \mathrm{C}$ for $24 \mathrm{~h}$. The specimens were taken out of the oven after $24 \mathrm{~h}$, and the initial dry weights of the specimens were measured to a precision of $0.001 \mathrm{~g}$. The specimens were then located in an immersed state in water absorption dishes at room temperature for $2,4,6,8$, $24,48 \mathrm{~h}$, and also up to the time they reached a constant weight. After each given time period, the specimens were removed from water, and their secondary wet weights were recorded. They were then put into water to calculate their wet-weights again as above. Water absorptions of specimens were calculated according to the following equation using the data corresponding to the dry and the wet weights.

Water absorption $=\frac{\text { wet weight-dry weight }}{\text { dry weight }} \times 100$

\subsubsection{Scanning Electron Microscopy (SEM)}

The SEM specimens were frozen by liquid Nitrogen and then fractured. The Fresh fractured surfaces of specimens were gold coated using a Spotter coater model K450X (Emich Co., England) and imaged using a TESCAN model WEGA II SEM microscope (Czech Republic). The SEM micrographs were then prepared using a voltage of $20 \mathrm{Kv}$.

Table 2. Extruding parameters for the wood flour/HDPE composites

\begin{tabular}{ccccccccccc}
\hline $\begin{array}{c}\text { Plastic } \\
\text { type }\end{array}$ & Die & 5 & 4 & 3 & 2 & 1 & $\begin{array}{c}\text { Screw } \\
\text { speed } \\
(\mathrm{rpm})\end{array}$ & $\begin{array}{c}\text { Screw } \\
\text { frequency } \\
(\mathrm{Hz})\end{array}$ & $\begin{array}{c}\text { Die } \\
\text { pressure } \\
(\mathrm{pv})\end{array}$ \\
\hline HDPE & 135 & 165 & 170 & 170 & 180 & 180 & 10 & 13.60 & 8 \\
\hline
\end{tabular}




\subsubsection{Dynamic Mechanical Thermal Analysis (DMTA)}

The DMTA specimen dimensions were $30 \times 10 \times 2 \mathrm{~mm}$. DMTA was performed using a Rheometric Scientific DMTA analyzer model triton tritec 2000 BMA (England) at flexural mode with a constant oscillation frequency of $1 \mathrm{~Hz}$. The natural fiber HDPE composites were scanned over a temperature range of -150 to $150^{\circ} \mathrm{C}$. The heating rate was $5^{\circ} \mathrm{C} / \mathrm{min}$ for all temperature scan tests. Storage modulus ( $\mathrm{E}^{\prime}$ ) and mechanical loss factor $(\tan \delta)$ were collected during the test and plotted versus temperature.

\subsubsection{Statistical analysis}

The test results were evaluated through factorial experiments in a completely randomized design using 5 replications for each formulation. To analyze the data, one-way analysis of variance (ANOVA) was employed, and Duncan's multiple range test (DMRT) was used to group the means. All comparisons were done at $95 \%$ confidence level.

\section{Results and Discussion}

\subsection{Fiber length measurement}

As observed in figure 1, there is a falling trend in fibers length from the non-recycled composite formulations to the four-time recycled ones. The results show that after the first recycling, fibers underwent dramatic length reductions due to the compression and shear stresses existing in different zones of extruder; the length of wood fibers in original HDPE composites was $463 \mu \mathrm{m}$, whereas it reduced to $326 \mu \mathrm{m}$ after the first recycling. Figure 1 also shows that the trend of fiber length reduction is much slower from the one-time recycled composites to the four-time recycled ones, being $218 \mu \mathrm{m}$.

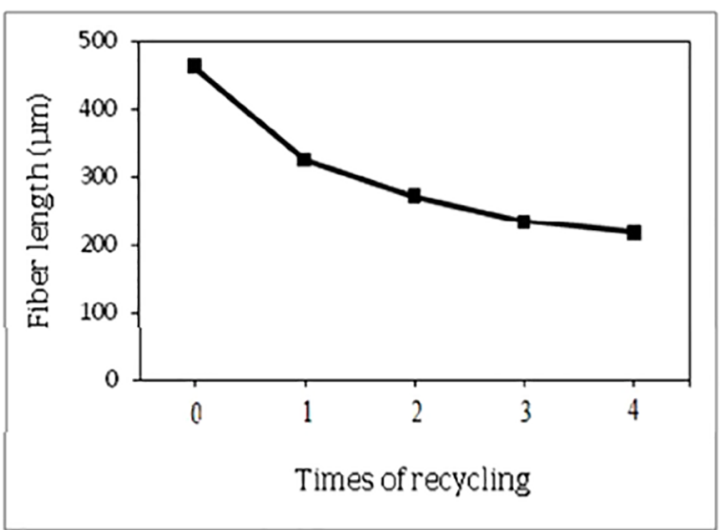

Figure 1. Fiber length of natural fiber HDPE composites prior to and after reprocessing.

\subsection{Static tests}

As seen in Figure 2, the bending strength of natural fiber HDPE composites decreased after reprocessing.
Such a result has also been reported by Beg and Pickering (2008). ${ }^{16,18}$ The results of analysis of variance obtained from bending strength test indicated that there was a statistically significant difference between the bending strength values of the studied composite prior to and after recycling at $95 \%$ confidence level. There were, as well, significant differences between the bending strength values of Rec1 samples and those of Rec 3 and Rec4, and likewise between Rec3 and Rec4 samples. The bending strength of natural fiber HDPE composites was 44.39 MPa prior to recycling, which decreased to 40.11 after the first recycling. This is possibly due to fiber fracture during Rec1, leading to reduced fiber length, and thus decreased bending strength. Similar results were also observed by Dickson et al. (2014) studying the effect of reprocessing on the mechanical properties of polypropylene reinforced with wood pulp, flax or glass fiber. ${ }^{15}$ The given property underwent very slight reductions in the second and third times of recycling, which can be attributed to the trace of fiber length reduction in the mentioned recycling times. SEM micrographs (figure 6) confirmed the authors' assumption showing that fiber length experienced a dramatic reduction after the first recycling time, but was very slightly reduced after the other times. As observed in fig. 2, there is a falling trend from the reference samples to Rec3 ones indicating that recycling (except for Rec4 samples showing a slight increase) led to reduced bending strength values. The increase in bending strength value of the studied composites after Rec4, which was almost similar to that of the Rec1 samples, is probably due to cross linking within polyethylene chains, which occurs in repeatedly recycled composites.

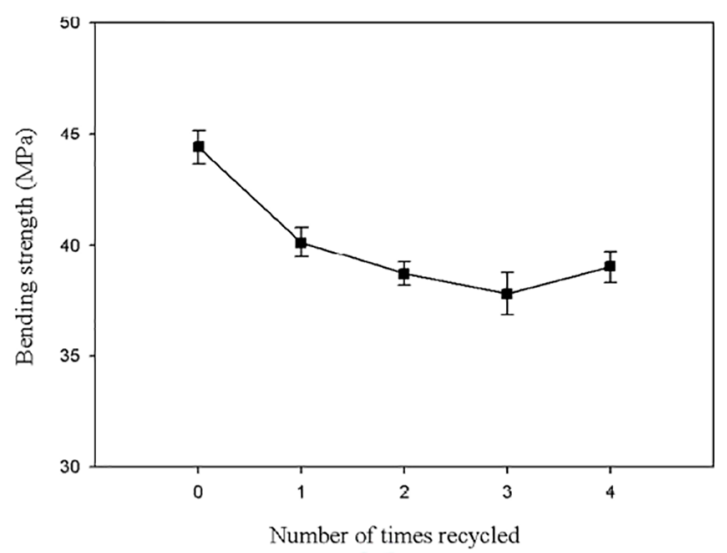

Figure 2. Bending strength of natural fiber HDPE composites prior to and after reprocessing.

Figure 3 exhibits the modulus of elasticity of the studied natural fiber HDPE composites prior to and after reprocessing, being $6728 \mathrm{MPa}$ prior to recycling, which decreased to 6368 MPa after the first recycling. From the literature, natural fibers serve as reinforcing components in natural fiber polymer composites, and fibers aspect ratio plays a crucial role in 
their reinforcing performance, thus the reduced fiber aspect ratio resulting from fiber fracture in the course of Rec1 may be the reason behind the mentioned reduction in the studied composites modulus of elasticity after Rec1. ${ }^{37,44}$ The given property showed very slight diminutions in the second and third times of recycling, which can be attributed to the trace of fiber length reduction in the mentioned recycling times. Considering modulus of elasticity, there was, as well, a decreasing trend from the reference samples to Rec3 ones indicating that recycling, except for Rec4 samples, resulted in decreased modulus of elasticity values in all the other recycled composites. ${ }^{16,18}$ The increase in modulus of elasticity value of the studied composites after Rec4 is not in agreement with the above-mentioned researches. This can be attributed to the polymer crosslinking, and hence growth of polymer crystalline sections. ${ }^{45}$ The mentioned phenomena along with better fiber-polymer interlocking due to crosslinking could result in the studied composites improved modulus of elasticity after Rec4. 12,13,37,46,47

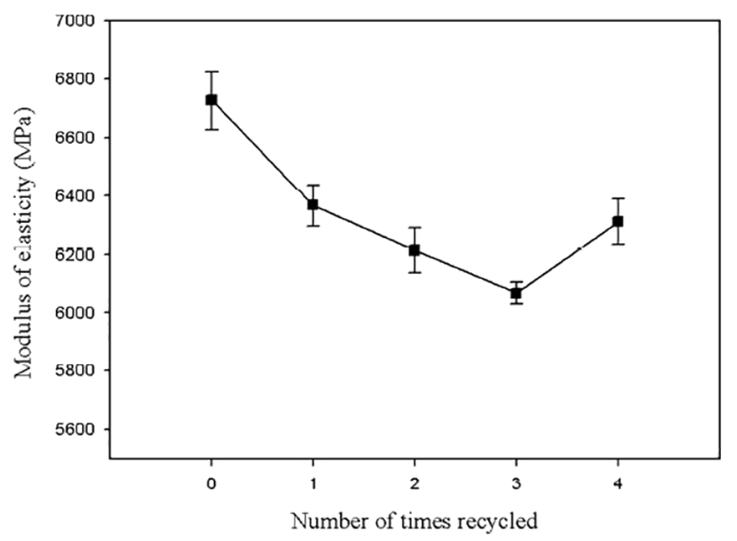

Figure 3. Modulus of elasticity of natural fiber HDPE composites prior to and after reprocessing.

The results derived from statistical analysis, also, revealed that there were significant differences between the modulus of elasticity of natural fiber HDPE composites prior to and after the first, second, third, and fourth time of recycling at 95\% confidence level.

Figure 4 displays the effects of the number of recycling times on the impact strength of natural fiber HDPE composites prior to and after recycling. The statistical analysis indicated that there were significant differences between the impact strength of natural fiber HDPE composites prior to and after the first, second, third, and fourth time of recycling. The impact strength value of reference natural fiber HDPE composites was $7.60 \mathrm{KJ} / \mathrm{m}^{2}$, which boosted to $8.69 \mathrm{KJ} / \mathrm{m}^{2}$ after the first recycling. During the first recycling, the length of particles is reduced as a result of mechanical attrition, leading to the enhanced polymer-fiber interlocking and mixture. Thus, the fibers surfaces are, to a higher extent, encapsulated by the molten HDPE polymer bringing about boosted interfacial adhesion. This, in turn, results in better stress transfer and so increased impact strength value. The given property slightly decreased after Rec 2 and Rec3, due to slight fiber length reductions, leading to reduced stress transfer rate, but again improved after Rec4. As stated above, cross linking is the dominant phenomenon during the fourth recycling step, which is why the impact strength of the studied samples increased. This is in agreement with the results reported by Tamboli et al. (2004) indicating that cross linking could lead to improved impact performance of polyethylene composites. ${ }^{45}$ Furthermore, statistically significant differences were observed between the impact strength values of Rec1 samples and those of Rec3 and Rec4, and as well between $\operatorname{Rec} 3$ and Rec4 samples. In a study on injection molding reprocessing of hemp fiber in recycled PP, Bourmaud et al. (2011) also observed an increase in impact strength value resulting from reprocessing..$^{40}$ From the literature, cross linking leads to improved impact performance of polyethylene composites. ${ }^{45}$ The variability of impact data for natural fiber composites is well known, and Sobczak et al. (2013) pointed out a number of apparently contradictory trends in impact strength in reviewing the effect of modifications on the fiber-matrix interface in natural fiber composites. Yet, The reported impact data on reprocessed natural fibers is relatively rare. ${ }^{48}$

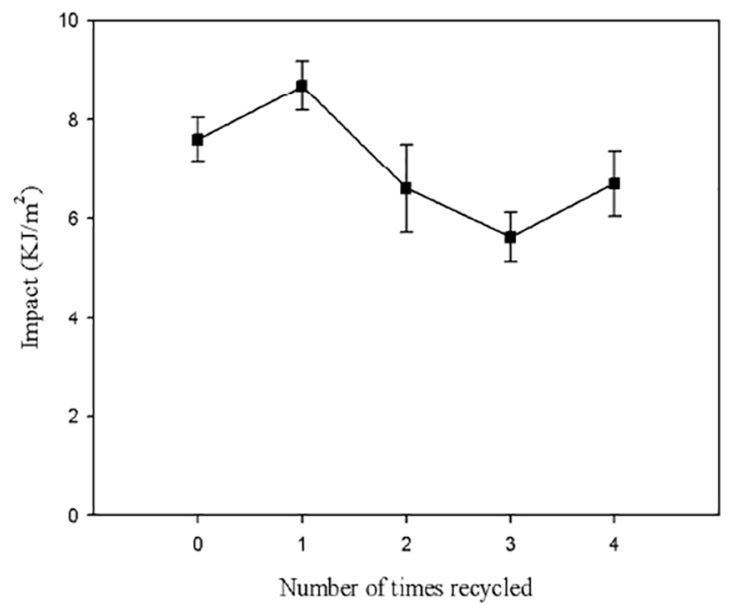

Figure 4. Impact strength of natural fiber HDPE composites prior to and after reprocessing.

\subsection{Physical properties}

As can be seen in figure 5, The maximal and minimal water absorption rates were pertaining to the original and four-time recycled natural fiber HDPE composites, respectively. The longer the studied samples were immersed in water, the higher their water absorption percentages increased. However, the increase in water absorption was terminated as the samples reached saturation point. The natural fiber HDPE composites, which were recycled more, possessed lower water absorption percentages and thus were 
saturated sooner compared to the lesser recycled or original counterparts. The reference samples had a larger fraction of voids and neat fibers, as compared to the recycled ones, resulting in their higher water absorption percentages. As a result of recycling, polymer ingresses and fills the pits existing in the cell walls and likewise the lumens of wood fibers, thereby reducing water absorption percentage in recycled samples. Furthermore, It can be assumed that the fibers wetted and encapsulated well by the molten polymer possess lower water absorption, which is why the Rec4 samples exhibited lowest water absorption percentages, as compared to the other samples.

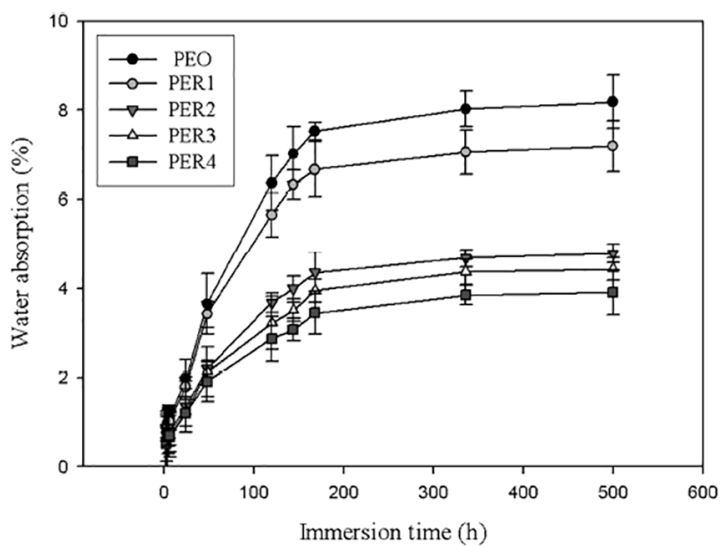

Figure 5. Water absorption of natural fiber HDPE composites prior to and after reprocessing.

On the other hand, the hemicelluloses present in natural fibers hold substantial hydroxyl groups making them the most hydrophilic constituents of wood plastic composites (WPC), and likewise the most susceptible to thermal degradation. From the literature reviewed, recycling leads to degradation and loss of WPCs hemicellulose fraction, leaving them more hydrophobic. That is why a lesser hydrophlilic nature was observed in the recycled samples. Studying on the water absorption/desorption of recycled natural fiber polymer composites, Tajvidi and Takemura (2010) reported that the concentration of hydroxyl groups and, in turn the hemicellulose fraction decreased as the number of recycling times increased. ${ }^{2}$ Thus, it is concluded that the chemical changes resulting from recycling can play a role in the water absorption rate of natural fiber polymer composites.

Lower fiber length brought about through recycling causes better dispersion and uniformity of the fiber distribution, which in turn lead to reduced water absorption. This stands as proof indicating why the water absorption of the studied composites reduced as the number of recycling times rose. It seems that the water absorption of natural fiber HDPE composites does not present as much of a problem when recycling is an issue.

\subsection{Scanning Electron Microscopy (SEM)}

Figure 6 shows the SEM images of natural fiber HDPE polymer composites prior to and after one to 4 times of recycling. As observed, fiber pull-out from the polymer matrix can be seen in recycled composites; the fibers are easily pulled out of the fractured surfaces of the studied composite formulations, leading to the appearance of voids in the mentioned surfaces. After the third recycling, several and by far larger voids are observed in the fractured surfaces of the given composites brought about by fiber pull-out due to poor interfacial adhesion, which resulted in the loss of mechanical properties. As observed in figure 6, fiber pullout is almost trace in the four-time recycled composites, which indicates a better fiber-polymer interfacial adhesion leading to improved mechanical properties for the mentioned composite formulations.

\subsection{Dynamic Mechanical Thermal Analysis (DMTA)}

Figure 7 displays the storage modulus spectrum of natural fiber HDPE composites at the oscillation frequency of $1 \mathrm{~Hz}$ as a function of temperature. To compare the effect of recycling on dynamic mechanical properties, the storage modulus curves of natural fiber HDPE composites were superposed. As can be observed, there is a reduction in the storage modulus due to recycling, and a major phase transition is seen in the studied materials. The transition temperature varied as different formulations of original and recycled materials were used. Under the phase transition temperature, the storage modulus values fall as the temperature increases. In the vicinity of glass transition temperature, a steep decline is observed, which indicates that the material is going through a glassy state to a rubber one. Over the whole range of temperature applied in this research, the Rec2 samples owned the minimal values of storage modulus. Rec1 and Rec 4 samples behaved in a fairly similar manner almost overlapping all over the temperature range. The phase transition temperature of the original composites was higher than that of the recycled counterparts. To note that it is difficult to determine a single phase transition temperature for all Composite formulations, since the transition actually occurs over a range of temperature, which is typical in semicrystalline polymers.

Figure 7 illustrates that all the recycled composites possessed a lower storage modulus as compared to the reference (original) natural fiber HDPE samples, which is due to the reduction of fibers length induced by the shear tensions present in the course of reprocessing (internal recycling). This comes as proof why the recycled composite formulations possessed a lower reinforcing efficiency, and in turn reduced mechanical properties, as compared to the reference counterparts. The reduced fibers length resulting from recycling can be easily observed in SEM images (figure 6). 

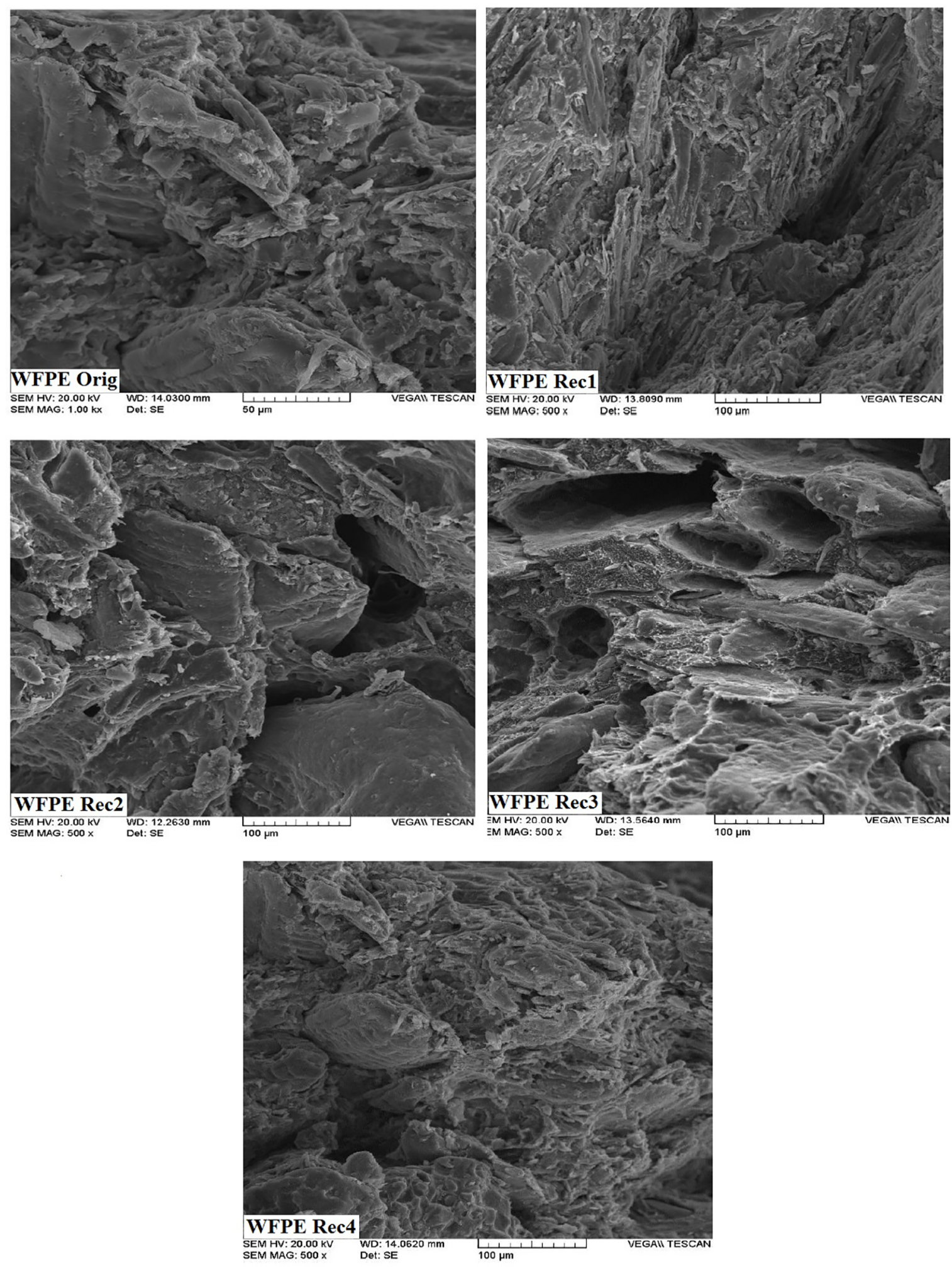

Figure 6. SEM micrographs of fractured surfaces of composites at $500 \mathrm{x}$ prior to and after processing. 


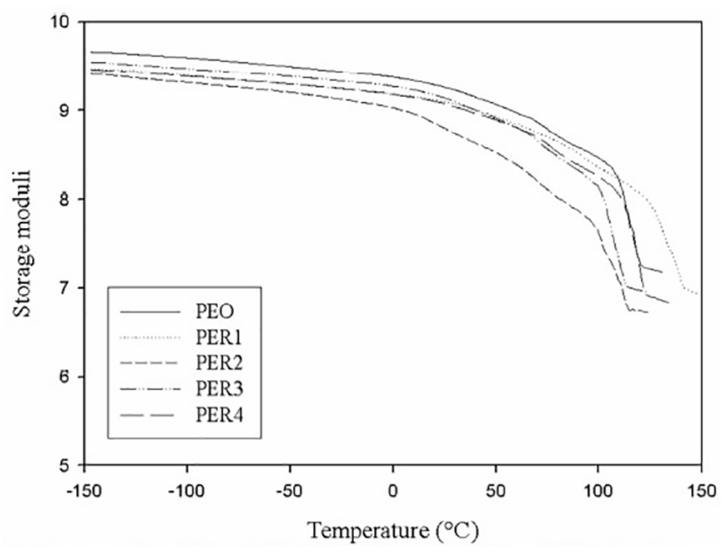

Figure 7. Storage modulus of natural fiber HDPE composites prior to and after reprocessing.

Figure 7, as well, reveals that there was a slight difference in the storage moduli of the Rec 2 and Rec 3 composites, confirmed by their respective fiber lengths, which are almost identical. As illustrated in Figure 7, there was a marked increase in the storage modulus, and hence improved reinforcement efficiency of Rec4 samples, as compared to that of Rec 1 and Rec 2 counterparts, which can be attributed to the cross linking phenomenon occurring in repeatedly recycled composites.

Figure 8 exhibits the spectrum of mechanical loss factor in natural fiber HDPE composites prior to and after recycling. Three main transitions can be easily observed. The first transition observed at about $60^{\circ} \mathrm{C}$ (in the original composites) is $\gamma$ transition. There is a similar trend for the original and recycled composites, but the location and intensity of the transitions differ. The $\gamma$ transition temperature for the original composites was higher than that of the recycled natural fiber HDPE formulations. The second transition occurred at a temperature range of $80-90{ }^{\circ} \mathrm{C}$, which is $\beta$ transition. The third transition, which occurs before melting, at about $140^{\circ} \mathrm{C}$ is called $\alpha$ transition.

As can be observed in figure 8, The spectra of mechanical loss factor $(\tan \delta)$ prior to and after recycling revealed an increase for all composites subjected to recycling. Higher tan $\delta$ of the recycled composite formulations clearly indicated that these formulations exhibited more viscous (dashpot-like) than elastic (spring-like) nature, as compared to the reference. ${ }^{24}$

Both of $\alpha$ and $\beta$ transitions had the same trend. The mentioned transitions of the Rec1 composites shifted to temperatures higher than those of the original and recycled ones. During the first recycling, the length of particles is reduced as a result of mechanical attrition, leading to the enhanced polymer-fiber interlocking and mixture. Thus, the fibers surfaces are, to a higher extent, encapsulated by the molten HDPE polymer bringing about improved interfacial adhesion. Therefore, polymer molecular motions are restricted, and in turn $\alpha$ and $\beta$ transition occur at higher temperatures in Rec1 samples, compared to those of all the

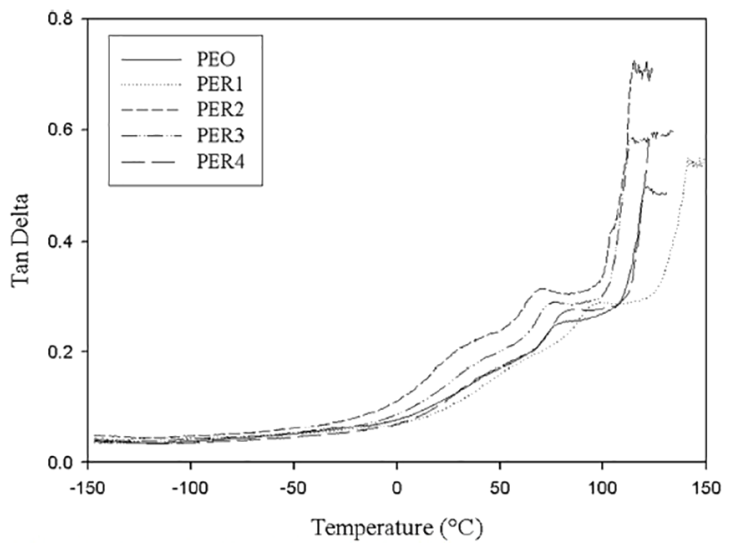

Figure 8. Mechanical loss factor $(\tan \delta$ ) of natural fiber HDPE composites prior to and after recycling.

other composite formulations. Figure 8 also shows that the $\alpha$ and $\beta$ transition of the Rec 2 and Rec 3 composites shifted to lower temperatures, as compared to the reference. The reduced fiber aspect ratio resulting from fiber fracture and/ or chain scission during Rec2 and Rec3 was possibly the dominant phenomenon accounting for the reduced reinforcing performance of fibers, which led to easier polymer molecular motions, and hence reduced temperatures of $\alpha$ and $\beta$ transitions. The given property showed very slight diminutions in the second and third times of recycling, which can be attributed to slightly reduced fiber length in the mentioned recycling times. The $\alpha$ and $\beta$ transitions of the Rec 4 composites were almost equivalent to those of the original samples, occurring at higher temperatures, as compared to those of the Rec2 and Rec 3 counterparts. This can be attributed to the cross linking phenomenon, which restricted the molecular mobility of the polymer chains leading to increased temperatures of $\alpha$ and $\beta$ transitions and the resultant delay in the melting point of Rec 4 composites.

Soccalingame et al. (2015) conducted a research on reprocessing of artificial UV-weatherd wood flour reinforced polypropylene and proffered that the static mechanical properties measured by tensile and impact tests underwent a "regeneration" phenomenon. Reprocessing induced a recovering of their initial properties. They attributed this phenomenon to the competition between chain scission and recombination mechanisms as well as a transfer of degraded chains from amorphous phases to crystalline phases. ${ }^{3}$ Moreover, dynamic mechanical spectrometry carried out by DMTA presented the same "regeneration" trend regarding the glass transition temperature $(T \alpha)$, which shifted to higher temperatures after reprocessing as related by Jansson et al. $(2003)^{49}$ and Luzuriaga et al. (2006). ${ }^{50}$

\section{Conclusions}

In this research, natural fiber HDPE composites were manufactured and then reprocessed up to four times in order 
to evaluate the effects of reprocessing (internal recycling) on the physical, mechanical as well as dynamic properties of natural fiber high-density polyethylene composites, and likewise to compare the mentioned properties with those of the original composites.

Doing so, the following conclusions could be obtained from the present research:

- The bending strength of the studied natural fiber HDPE composites exhibited a falling trend (except for Rec4 samples) after recycling. It was concluded that this was possibly due to fiber fracture during recycling, resulting in reduced fiber length, and thus decreased bending strength values. The increase in bending strength of the Rec4 samples was attributed to cross linking brought about by repeated recycling.

- The composites modulus of elasticity possessed the same trend as bending strength prior to and after reprocessing. The reduced fiber aspect ratio due to fiber fracture during Rec1 could account for the reduced modulus of elasticity after Rec1. It was concluded that the molecular weight of polymer enhanced though crosslinking, and in turn growth of polymer crystalline sections. The mentioned phenomena along with better fiberpolymer entanglement due to crosslinking could result in higher modulus of elasticity after Rec4.

- Unlike the above-mentioned properties, the impact strength of the given composites enhanced after Rec1 where the fractured fibers were better encapsulated by the polymer bringing about better interfacial adhesion, and in turn better stress transfer, which resulted in higher impact strength values. It was concluded that cross linking could be the dominant phenomenon during the fourth recycling step accounting for the corresponding improvement of the impact strength value.

- The more the composited were recycled, the lower water absorption potential they exhibited. The molten polymer ingresses the voids existing within the composite matrix during recycling, thereby reducing its water absorption percentage. The Rec4 samples showed the lowest water absorption percentages due to better fiber wetting and encapsulation. It was also proffered that recycling could lead to the degradation of hemicellulose in WPCs, leaving them less hydrophilic.

- DMTA analysis revealed that all the recycled composites possessed a lower storage modulus due to fiber length reduction caused by the shear tensions during reprocessing. This, in turn, brought about lower reinforcing efficiency for the recycled composites. There was a considerable increase in the storage modulus of Rec4 samples, which was attributed to the cross linking phenomenon resulting in the improved mechanical properties of Rec4 samples observed in this study.

- The spectra of mechanical loss factor $(\tan \delta)$ revealed an increase in mechanical loss factor for all composites subjected to recycling. Higher tan $\delta$ of the recycled composite clearly indicated that these formulations exhibited more viscous than elastic nature. Both $\alpha$ and $\beta$ transitions had the same trend. The mentioned transitions of the Rec1 composites shifted to higher temperatures due to better polymer-fiber interlocking and encapsulation, which, in turn brought about improved interfacial adhesion. Thus, the molecular motions of polymer chains were restricted, shifting $\alpha$ and $\beta$ transitions to higher temperatures. The $\alpha$ and $\beta$ transitions of the Rec4 composites were almost similar to those of the reference. This was attributed to the cross linking phenomenon, which restricted the molecular mobility of the polymer chains leading to increased temperatures of $\alpha$ and $\beta$ transitions and the resultant delay in the melting point of Rec 4 composites.

\section{Acknowledgements}

The authors would like to express their appreciation to the department of wood and paper science and technology, University of Tehran and Petrochemical Research and Technology Company (NPC-rt), National Petrochemical Company (NPC) for the kind supports of this project.

This research was funded by the authors and also the department of wood and paper science and technology, University of Tehran, Iran.

\section{References}

1. Muthuraj R, Misra M, Mohanty AK. Studies on mechanical, thermal, and morphological characteristics of biocomposites from biodegradable polymer blends and natural fibers. In: Misra M, Pandey JK, Mohanty AK, eds. Biocomposites Design and Mechanical Performance. Cambridge: Woodhead Publishing; 2015. p. 93-140.

2. Tajvidi M, Takemura A. Recycled Natural Fiber Polypropylene Composites: Water Absorption/Desorption Kinetics and Dimensional Stability. Journal of Polymers and the Environment. 2010;18(4):500-509.

3. Soccalingame L, Perrin D, Bénézet JC, Mani S, Coiffier F, Richaud E, et al. Reprocessing of artificial UV-weathered wood flour reinforced polypropylene composites. Polymer Degradation and Stability. 2015;120:313-327. DOI: 10.1016/j. polymdegradstab.2015.07.013

4. Soccalingame L, Bourmaud A, Perrin D, Bénézet JC, Bergeret A. Reprocessing of wood flour reinforced polypropylene composites: Impact of particle size and coupling agent on composite and particle properties. Polymer Degradation and Stability. 2015;113:72-85. 
5. Guerrica-Echevarría G, Eguiazábal JI, Nazábal J. Effects of processing conditions on the properties of unfilled and talc-filled polypropylene. Polymer Degradation and Stability. 1996;53(1):1-8.

6. Balatinecz JJ, Sain MM. The influence of recycling of wood fiber - Plastic composites. Macromolecular Symposia. 1998;135(1):167-173.

7. Canevarolo SV. Chain scission distribution function for polypropylene degradation during multiple extrusions. Polymer Degradation and Stability. 2000;70(1):71-76.

8. Xiang Q, Xanthos M, Mitra S, Patel SH, Guo J. Effects of melt reprocessing on volatile emissions and structural/rheological changes of unstabilized polypropylene. Polymer Degradation and Stability. 2002;77(1):93-102

9. Martins MH, De Paoli MA. Polypropylene compounding with post-consumer material: II. Reprocessing. Polymer Degradation and Stability. 2002;78(3):491-495.

10. Da Costa HM, Ramos VD, Rocha MCG. Rheological properties of polypropylene during multiple extrusion. Polymer Testing. 2005;24(1):86-93.

11. Meran C, Ozturk O, Yuksel M. Examination of the possibility of recycling and utilizing recycled polyethylene and polypropylene. Materials \& Design. 2008;29(3):701-705.

12. Kazemi-Najafi S, Mostafazadeh-Marznaaki M, Chaharmahali M, Tajvidi M. Effect of Thermomechanical Degradation of Polypropylene on Mechanical Properties of WoodPolypropylene Composites. Journal of Composite Materials. 2009;43(22):2543-2554.

13. Bahlouli N, Pessey D, Raveyre C, Guillet J, Ahzi S, Dahoun A, et al. Recycling effects on the rheological and thermomechanical properties of polypropylene-based composites. Materials \& Design. 2012;33:451-458.

14. Teuber L, Osburg VS, Toporowski W, Militz H, Krause A. Wood polymer composites and their contribution to cascading utilization. Journal of Cleaner Production. 2016;110:9-15. DOI: $10.1016 /$ j.jclepro.2015.04.009

15. Dickson AR, Even D, Warnes JM, Fernyhough A. The effect of reprocessing on the mechanical properties of polypropylene reinforced with wood pulp, flax or glass fibre. Composites Part A: Applied Science and Manufacturing. 2014;61:258-267.

16. Beg MDH, Pickering KL. Reprocessing of wood fibre reinforced polypropylene composites. Part I: Effects on physical and mechanical properties. Composites Part A: Applied Science and Manufacturing. 2008;39(7):1091-100.

17. Lopez JP, Girones J, Mendez JA, Puig J, Pelach MA. Recycling Ability of Biodegradable Matrices and Their Cellulose-Reinforced Composites in a Plastic Recycling Stream. Journal of Polymers and the Environment. 2012;20(1):96-103.

18. Beg MDH, Pickering KL. Reprocessing of wood fibre reinforced polypropylene composites. Part II: Hygrothermal ageing and its effects. Composites Part A: Applied Science and Manufacturing. 2008;39(9):1565-1571.

19. Bakkal M, Bodur MS, Berkalp OB, Yilmaz S. The effect of reprocessing on the mechanical properties of the waste fabric reinforced composites. Journal of Material Processing Technology. 2012;212(11):2541-2548.
20. Ausias G, Bourmaud A, Coroller G, Baley C. Study of the fibre morphology stability in polypropylene-flax composites. Polymer Degradation and Stability. 2013;98(6):1216-1224.

21. Sarabi MT, Behravesh AH, Shahi P, Soury E. Reprocessing of Extruded Wood- Plastic Composites; Mechanical Properties. Journal of Biobased Materials and Bioenergy. 2012;6(2):221-229.

22. Kraiem D, Pimbert S, Ayadi A, Bradai C. Effect of low content reed (Phragmite australis) fibers on the mechanical properties of recycled HDPE composites. Composites Part B: Engineering. 2013;44(1):368-374.

23. Morán J, Alvarez V, Petrucci R, Kenny J, Vazquez A. Mechanical properties of polypropylene composites based on natural fibers subjected to multiple extrusion cycles. Journal of Applied Polymer Science. 2007;103(1):228-237.

24. Tajvidi M, Falk RH, Hermanson JC. Effect of natural fibers on thermal and mechanical properties of natural fiber polypropylene composites studied by dynamic mechanical analysis. Journal of Applied Polymer Science. 2006;101(6):4341-4349.

25. Kurvilla J, Sabu T, Pavithran C. Dynamic Mechanical Properties of Short Sisal Fiber Reinforced Low Density Polyethylene Composites. Journal of Reinforced Plastics and Composites. 1993;12(2):139-155.

26. Kim TJ, Lee YM, Im SS. The preparation and characteristics of low-density polyethylene composites containing cellulose treated with cellulase. Polymer Composites. 1997;18(3):273-280.

27. Rodríguez-Pérez MA, Rodríguez-llorente S, De Saja JA. Dynamic mechanical properties of polyolefin foams studied by DMA techniques. Polymer Engineering \& Science. 1997;37(6):959-965.

28. Simonsen J, Jacobsen R, Rowell R. Wood-fiber reinforcement of styrene-maleic anhydride copolymers. Journal of Applied Polymer Science. 1998;68(10):1567-1573.

29. Reussmann T, Mieck KP. Dynamic-mechanical Analysis of Natural Fiber Reinforced Plastics. Advanced Engineering Materials. 1999;1(2):140-141.

30. Menard KP. Dynamic Mechanical Analysis: A Practical Introduction. Boca Raton: CRC Press; 1999. 208 p.

31. Landel RF, Nielsen LE. Mechanical Properties of Polymers and Composites. $2^{\text {nd }}$ ed. New York: Marcel Dekker; 1993. 580 p.

32. Fonseca-Valero C, Ochoa-Mendoza A, Arranz-Andrés J, GonzálezSánchez C. Mechanical recycling and composition effects on the properties and structure of hardwood cellulose-reinforced high density polyethylene eco-composites. Composites Part A: Applied Science and Manufacturing. 2015;69:94-104.

33. Li R, Ye L, Mai YW. Effect of polyethylene particle geometry on mechanical properties of compression molded wood-polyethylene composites. Plastic, Rubber and Composites Processing and Applications. 1997;26(8):368-371.

34. Jayaraman K, Bhattacharyya D. Mechanical performance of woodfibre-waste plastic composite materials. Resources Conservation and Recycling. 2004;41(4):307-319.

35. La Mantia FP, Morreale M. Mechanical properties of recycled polyethylene ecocomposites filled with natural organic fillers. Polymer Engineering \& Science. 2006;46(9):1131-1139. 
36. Lei Y, Wu Q, Yao F, Xu Y. Preparation and properties of recycled HDPE/natural fiber composites. Composites Part A: Applied Science and Manufacturing. 2007;38(7):1664-1674.

37. Adhikary KB, Pang S, Staiger MP. Dimensional stability and mechanical behaviour of wood-plastic composites based on recycled and virgin high-density polyethylene (HDPE). Composites Part B: Engineering. 2007;39(5):807-315.

38. Arbelaiz A, Fernández B, Ramos JA, Retegi A, Llano-Ponte R, Mondragon I. Mechanical properties of short flax fibre bundle/ polypropylene composites: Influence of matrix/fibre modification, fibre content, water uptake and recycling. Composite Science and Technology. 2005;65(10):1582-1592.

39. Bourmaud A, Baley C. Investigations on the recycling of hemp and sisal fibre reinforced polypropylene composites. Polymer Degradation and Stability. 2007;92(6):1034-1045.

40. Bourmaud A, Le Duigou A, Baley C. What is the technical and environmental interest in reusing a recycled polypropylenehemp fibre composite? Polymer Degradation and Stability. 2011;96(10):1732-1739.

41. Srebrenskoska V, Gaceva GB, Avella M, Errico ME, Gentile G. Recycling of polypropylene-based eco-composites. Polymer International. 2008;57(11):1252-1257.

42. Kurniawan D, Kim BS, Lee HY, Lim JY. Effects of repetitive processing, wood content, and coupling agent on the mechanical, thermal, and water absorption properties of wood/polypropylene green composites. Journal of Adhesion Science and Technology. 2013;27(12):1301-1312.
43. Shahi P, Behravesh AH, Daryabari SY, Lotfi M. Experimental investigation on reprocessing of extruded wood flour/HDPE composites. Polymer Composites. 2012;33(5):753-763.

44. Guo Q, Cheng B, Sain MKM, Knudson R, Deng J, Alemdar A. Performance of long Canadian natural fibers as reinforcements in polymers. Journal of Reinforced Plastics Composites. 2010;29(21):3197-3207.

45. Tamboli SM, Mhaske ST, Kale DD. Crosslinked polyethylene. Indian Journal of Chemical Technology. 2004;11(6):853-864.

46. Sadrmohaghegh C, Scott G. Effect of reprocessing on polymers: Part II-Polypropylene. Polymer Degradation and Stability. 1981;3(5):333-340.

47. Migneault S, Koubaa A, Erchiqui F, Chaala A, Englund K, Wolcott MP. Effects of processing method and fiber size on the structure and properties of wood-plastic composites. Composites Part A: Applied Science and Manufacturing. 2009;40(1):80-85.

48. Sobczak L, Brüggemann O, Putz RF. Polyolefin composites with natural fibers and wood- modification of the fiber/ filler-matrix interaction. Journal of Applied Polymer Science. 2013;127(1):1-17.

49. Jansson A, Möller K, Gevert T. Degradation of post-consumer polypropylene materials exposed to simulated recycling-mechanical properties. Polymer Degradation Stability. 2003;82(1):37-46.

50. Luzuriaga S, Kovárova J, Fortelný I. Degradation of pre-aged polymers exposed to simulated recycling: Properties and thermal stability. Polymer Degradation and Stability. 2006;91(6):12261232. 